\section{PERFIL DA UTILIZAÇÃO DAS TERAPIAS ALTERNATIVAS/ COMPLEMENTARES DE SAÚDE DE INDIVÍDUOS ORIUNDOS DO SISTEMA COMPLEMENTAR DE SAÚDE}

\section{PROFILE OF PATIENTS USING \\ ALTERNATIVE/COMPLEMENTARY THERAPIES BY INDIVIDUALS FROM SUPPLEMENTAL HEALTH SYSTEM}

\section{RESUMO}

Estudo do tipo survey quantitativo com o objetivo de descrever o perfil de utilização das terapias alternativas/complementares (TAC). A amostra por conveniência não probabilística foi composta por 237 pacientes de uma instituição privada de médio porte, da cidade de São Paulo, Brazil. A maioria da amostra foi constituída por adultos (57\%) e do sexo feminino (61\%). A utilização de algum tipo de TAC foi relatada por $83 \%$ dos entrevistados, com predominância da acupuntura. A experiência anterior positiva e o fato de ser um tratamento não medicamentoso foram os motivos mais citados para utilização das TAC. A orientação específica de um profissional de saúde foi pouco indicada. A perspectiva de novas formas de tratamento aliada às observações de resultados satisfatórios figuraram como importantes razões para adesão a este tipo de tratamento. A solução da queixa clínica, bem como o custo elevado foram apontados pela amostra estudada, como principais motivos para a não continuidade de uso das terapias complementares.

\section{PALAVRAS CHAVE}

Enfermagem.

Terapias Complementares.

Acupuntura.

Promoção da Saúde.
Maria Fernanda Zorzi Gatti

- Enfermeira Coordenadora da área de Emergência do Hospital Samaritano.

Eliseth Ribeiro Leão

- Pesquisadora Instituto Israelita de Ensino e Pesquisa Albert Einstein Hospital Israelita Albert Einstein

Maria Julia Paes Silva

- Livre Docente da Escola de Enfermagem da Universidade de São Paulo

Celice Romero Aquino

- Coordenadora Adjunta de qualidade do Hospital Maternidade Santa Joana e Pro Matre Paulista

C ORRESP ONDENTE

Maria Julia Paes Silva

Departamento Médico-Cirúrgico Escola de Enfermagem da USP

Av. Dr. Enéas de Carvalho Aguiar, 419 São Paulo - Brasil

E-M A I L

juliaps@usp.br

Recebido: 11/04/2014

Aprovado: 03/02/2015 


\begin{abstract}
Survey study quantitative to describe the profile of use of Complementary and Alternative Medicine (CAM). Not probabilistic convenience sample consisted of 237 patients of a midsize private institution of São Paulo, Brazil. The majority of the sample consisted of adults (57\%) and most women in this sample (61\%). The use of some modality of CAM was reported by $83 \%$ of respondents, acupuncture predominantly. The previous positive experience and also because it is a non-drug treatment were the most cited reasons for using the CAM. Specific guidance of a health professional about CAM was subindicated. The perspective related of new forms of treatment, and the observations of satisfactory results were important reasons for adherence to this modality of treatment. The resolutions of clinical complaints (healing), as well as the high cost were appointed by the sample studied as major reasons for not continuing utilization of complementary therapies.
\end{abstract}

KEYWORDS: Nursing. Complementary Therapies. Acupuncture. Health Promotion.

\section{INTRODUÇÃO}

A terminologia Terapias Alternativas Complementares (TAC), ou integrativas, não é mundialmente aceita. O que é definido como "alternativo" ou "complementar" em um país, pode ser considerado oficial, convencional ou tradicional em outro. Exemplo, a acupuntura na China.

Segundo a Organização Mundial da Saúde OMS, medicina alternativa complementar é o que não faz parte da tradição de um país e, desta forma, não é integrado ao sistema de saúde prevalente ${ }^{1}$.

O termo "alternativo" pode ser entendido como "outra metodologia" ou "outro modo" utilizado de se fazer um tratamento, em detrimento do convencional; já o termo "complementar" é compreendido como um facilitador no gerenciamento de sintomas de uma doença, que pode melhorar a qualidade de vida do paciente durante seu tratamento, realizado de maneira "padrão", naquele contexto ${ }^{2}$.

Ainda, o termo "medicina complementar alternativa" pode ser substituído por "terapia integrativa", definido como uma combinação da medicina tradicional e alternativa, buscando atender as necessidades próprias de cada indivíduo ${ }^{3}$.

Fato é que a maioria das terapias utilizadas em práticas complementares na atualidade surgiu na antiguidade e foi "redescoberta" pelo mundo ocidental somente nessas últimas décadas, graças ao movimento de mudança paradigmática de compreensão de mundo e de realidade ${ }^{4}$.
É sabido também que o crescimento acelerado e não planejado da população mundial colaborou para as condições subumanas de sobrevivência e de carência por um sistema de saúde eficiente 5 . Esse é um dos motivos que contribui para que, na prática hospitalar, comumente encontremos pacientes que utilizam métodos alternativos-complementares em adição ao seu tratamento convencional ${ }^{6}$.

O crescimento desse uso nos últimos 15 anos indiscutivelmente tem despertado a atenção entre os profissionais da saúde e provocado mudanças na área econômica e social ${ }^{7}$. A própria OMS, no final da década de 70, criou o Programa de Medicina Tradicional, com o objetivo de formular diretrizes de incentivo à criação de políticas públicas para o uso consciente e integrado da medicina tradicional e complementaralternativa nos sistemas nacionais de saúde.

Ainda hoje, porém, não há um consenso para a classificação das TAC. Nos EUA, o National Center for Complementary and Alternative Medicine (NCCAM) propõe uma divisão em cinco categorias':

- produtos naturais: incluindo plantas medicinais, vitaminas, minerais, suplemento alimentar e probióticos;

- intervenção mente-corpo: meditação, ioga, acupuntura, relaxamento, oração, hipnoterapia, terapia psicológica, arte terapia;

- manipulação ou método baseado no corpo: técnicas de massagem, quiropraxia; 
- terapias energéticas: Qi Gong, reiki, reflexologia, ou baseada em bioeletromagnética;

- sistema médico alternativo: homeopatia, medicina tradicional chinesa, medicina Ayurvédica.

A escolha por essas terapias não convencionais parece ser um fator significativo no tratamento de pacientes em países pobres e em desenvolvimento ${ }^{8}$. Pouco se sabe, porém, sobre o uso dessas práticas em pessoas com poder aquisitivo maior.

Para muitos pacientes, as TAC são percebidas como menos agressivas. No entanto, para aumentar o potencial de seu uso, uma gama de questões relacionadas com a política, segurança, eficácia, acesso e uso, precisam ser revistas ${ }^{4}$.

No município de São Paulo o aumento da utilização das TAC tem sido percebido por $87,6 \%$ dos médicos, sendo considerados resultados positivos em $61,5 \%$ dos casos e $42,8 \%$ desses profissionais acredita obter o mesmo efeito que a terapia convencional ${ }^{9}$.

O Ministério da Saúde (MS) afirma que as TAC atuam no campo da prevenção, promoção, manutenção e recuperação da saúde, tendo em vista seu modelo de atenção humanizada e centrada na integralidade do indivíduo, o que contribui para o fortalecimento dos princípios fundamentais do Sistema Único de Saúde - SUS ${ }^{10}$. Com o reconhecimento dos benefícios das TAC foi aprovada a portaria $\mathrm{n}^{\circ} 971$ que criou a Política Nacional de Práticas Integrativas e Complementares (PNPIC) no SUS, em 03 de maio de $2006^{11}$. Além disso, as TAC encontram-se na Agenda Nacional de Prioridades de Pesquisa em Saúde (ANPPS) do MS ${ }^{12}$.

Importante ressaltar que as instituições de ensino da saúde não têm apresentado projetos pedagógicos que privilegiem o olhar holístico (holos = todo), pois a despeito de lidarmos com seres humanos, os processos de trabalho contribuem para que as atividades sejam realizadas de forma fragmentada, despersonalizando a pessoa que é cuidada 9 .

Revisão integrativa da literatura avaliou 15 estudos que demonstram o interesse crescente sobre a temática das Terapias complementares, todavia de difícil percepção na aplicação das mesmas pelos enfermeiros no plano de cuidados. Os autores reforçam ainda a importância da divulgação e sensibilização quanto à educação dos profissionais de saúde, especialmente os Enfermeiros, em relação às Práticas Integrativas e Complementares $^{13}$.

Apesar da política pública instituída no país e da busca dos próprios pacientes pelas terapias complementares, existe ainda uma escassez de trabalhos científicos que expliquem sua utilização. Pouco se sabe sobre o perfil de quem as utiliza em nosso meio. Os estudos nacionais existentes se relacionam à percepção de estudantes ${ }^{1}$, dos profissionais da área da saúde ${ }^{14-15} \mathrm{e}$ de um número restrito de pacientes atendidos no sistema único de saúde ${ }^{16}$. Não sabemos como e se tem sido utilizadas por usuários do sistema de saúde suplementar. Sabe- se que no serviço público os pacientes têm acesso seja ao tratamento convencional ou complementar, no tempo e disponibilidade de cada serviço. Diferentemente, daqueles que podem usufruir de uma gama maior de serviços e recursos em saúde, mediante cobertura dos convênios, ou mesmo em caráter particular.

Este estudo busca investigar, portanto, a utilização das terapias complementares e sua efetividade, por indivíduos que não buscam atendimento em instituições públicas e sua efetividade. Os achados do estudo podem contribuir para conhecer o perfil de utilização para além dos já descritos na literatura, bem como poderá ser útil como subsidio para aumentar a oferta de serviços nessa área em instituições privadas e promover reflexões visando uma maior integração das terapias complementares na assistência à saúde.

\section{OBJETIVOS}

Conhecer o perfil de pacientes de um hospital privado quanto às terapias complementares no que se refere a: quais terapias são adotadas, os motivos de sua utilização, os resultados obtidos e as razões para a manutenção e/ou ao abandono das mesmas. 


\section{METODOLOGIA}

Trata-se de um estudo descritivo-exploratório, tipo survey, com abordagem quantitativa, conduzido de dezembro de 2009 a maio de 2010, junto a pacientes que se utilizam de exames e internações de um hospital privado, de médio porte, da cidade de São Paulo, no qual as terapias complementares são realizadas de forma isolada por alguns profissionais qualificados e inexiste um serviço específico que as ofereça regularmente.

A amostra por conveniência não probabilística, extraída da mala direta da instituição, foi constituída por 237 participantes que aderiram voluntariamente à pesquisa.

Após aprovação do Comitê de Ética e Pesquisa (CEP) do Hospital de estudo (aprovação 22/07), a coleta de dados foi realizada pelas pesquisadoras mediante o encaminhamento de uma Carta Convite, do Termo de Consentimento Livre e Esclarecido (TCLE) e do Questionário para atender ao objetivo proposto, elaborado pelas pesquisadoras que integram o Grupo de Pesquisa: Estudo das Práticas Alternativas e Complementares de Saúde, cadastrado no Conselho Nacional de Pesquisa (CNPq), filiado a uma universidade pública estadual.

Todos os documentos foram enviados via correio, em formato carta resposta. Os custos de impressão em gráfica e postagem foram subsidiados pelo convênio de pesquisa firmado entre o referido Hospital e a universidade pública de São Paulo.

As variáveis estudadas foram: terapias complementares utilizadas pelos participantes, motivos de sua utilização, avaliação dos resultados obtidos e as razões para a manutenção e/ou ao abandono das mesmas.

Os dados foram inseridos em banco de dados em Programa Excell e submetidos posteriormente à análise estatística descritiva.

\section{RESULTADOS E DISCUSSÃO}

Embora tenham sido postadas quatro mil e quinhentas correspondências 284 (6,3\%) retornaram lacradas com a justificativa do correio de endereço não encontrado, mudança de endereço ou de destinatário falecido. Duzentos e trinta e sete questionários retornaram respondidos e com respectivos TCLE assinados. Embora o fato dos respondentes corresponder a 5,6\% do total de envio e constitua a limitação mais significativa deste estudo, por não permitir generalizações, não desmerece o valor dos achados, tendo em vista não existir estudo semelhante conduzido em nosso meio e com essa população.

Não temos como saber as razões para a baixa adesão aos questionários, podemos inferir que a não utilização das terapias complementares pelos pacientes da instituição, destinatários não localizados cujas correspondências não retornaram, a falta de interesse em participar do estudo ou ainda, por se tratar de clientela já mais habituada aos meios eletrônicos de comunicação possam ter influenciado. $O$ fato de ter que postar os formulários em uma caixa de correio na atualidade parece não constituir o modo mais prático, principalmente ao considerarmos a dinâmica de vida tão corrida adotada em uma grande cidade. A disponibilidade de tempo, portanto, também é uma variável que pode ter interferido na taxa de retorno.

A principal desvantagem da utilização de questionários em pesquisa apontada por diversos autores é o baixo retorno de respostas ${ }^{17}$, que também é observada mesmo quando se lança mão desta técnica de coleta de dados via web ${ }^{18}$.

A maioria dos respondentes foi do sexo feminino $(61,1 \%)$, com faixa etária predominante entre 41 e 70 anos (57\%), e 70\% com nível superior de instrução ou pós-graduação.

Do total de participantes, $83 \%$ já utilizaram terapias complementares com predominância da acupuntura, conforme tabela 1. A acupuntura é uma prática já consagrada e profissionalizada para a qual a literatura já disponibiliza evidências científicas ${ }^{19}$. Por outro lado, trata-se de uma prática milenar, abordada nos meios de comunicação de massa, o que leva também a ser mais conhecida por maior número de indivíduos na população em geral. 
Tabela 1. Terapias complementares utilizadas pelos participantes da pesquisa. São Paulo 2009-2010.

\begin{tabular}{lc}
\hline Tipo de TAC utilizada & Total \\
\hline Acupuntura & 148 \\
\hline Floral & 94 \\
\hline Fitoterapia & 63 \\
\hline Yoga & 56 \\
\hline Reiki & 48 \\
\hline Meditação & 41 \\
\hline Auriculoterapia & 38 \\
\hline Massoterapia & 38 \\
\hline Reflexologia & 36 \\
\hline Hidroterapia & 33 \\
\hline Cromoterapia & 28 \\
\hline Antroposofia & 24 \\
\hline Quiropraxia & 21 \\
\hline Aromaterapia & 15 \\
\hline Iridologia & 10 \\
\hline Outras* & 10 \\
\hline Toque Terapêutico & 5 \\
\hline Geoterapia & 2 \\
\hline
\end{tabular}

* Homeopatia, Pilates, Fisioterapia, Ortomolecular

Nossos achados apontam para diferenças de perfil entre os participantes e os usuários do SUS quanto ao tipo de terapia complementar utilizada. A utilização de chás (50\%), fitoterapia (22,7\%) e cura espiritual $(22,7 \%)$ prevaleceram quando da análise de uma comunidade atendida pelo Programa de Saúde da Família ${ }^{16}$; e novamente o uso de chás medicinais foi o mais apontado (82\%) em população submetida à quimioterapia e atendida pelo Sistema Único de Saúde (SUS) ${ }^{20}$, ao passo que na nossa amostra, além de um número maior de terapias indicadas, a acupuntura seguido da terapia floral foram os maiores destaques.

Quando indagados sobre quem fez a indicação de uso, os profissionais de saúde correspondem a $29 \%$ das mesmas, o que é preocupante, pensarmos que menos de um terço da indicação de práticas voltadas à saúde, não são realizadas por profissionais qualificados. Estudo anterior também destacou que a minoria utiliza as TAC com acompanhamento de um profissional de saúde ${ }^{16}$.
A rede social de pacientes formada por amigos, vizinhos, familiares e profissionais, no acesso e apoio para o uso de TAC tem se mostrado ser de grande influência para pacientes em tratamentos convencionais ${ }^{21}$.

Frente a isso urge o investimento em ampliar o conhecimento do profissional de saúde em relação a TAC, como aspecto fundamental para $o$ atendimento da população com maior segurança. São necessários ainda investimentos em estudos sobre eficácia das TAC e em programas educacionais para que os profissionais de saúde estejam aptos a transmitir aos pacientes uma possibilidade real e segura de tratamento ${ }^{13,16,21}$.

Em relação à adesão ou não adesão a essas terapêuticas, $o$ fato de ser uma nova alternativa para $o$ tratamento de enfermidades (82\%), por terem observado resultados positivos anteriormente (27\%) e por se tratar de um tratamento não medicamentoso (26\%) foram os motivos mais citados para utilização da TAC. Os resultados ainda foram julgados como bons e ótimos por $78 \%$ dos participantes.

Por outro lado, os motivos relatados para não mais utilizarem esse tipo de tratamento se relacionaram ao fato do problema ter sido solucionado (35\%) ou pelo custo elevado de alguns tipos de TAC (32\%), as quais não foram especificadas.

Revisão de 32 artigos sobre o tema evidenciou, que além dos motivos já citados pelos participantes em nossa pesquisa, outros aspectos devem ser considerados quando se trata de investigar as razões pelas quais o paciente opta ou não pelo uso da TAC. Questões relacionadas à insatisfação com o tratamento médico convencional, a busca por alívio dos sintomas das doenças, a disponibilidade e acesso e o tempo de tratamento com a biomedicina são fatores que podem influenciar nessa decisão, bem como o preparo do terapeuta e a relação interpessoal mediada pela comunicação do profissional com o paciente ${ }^{22}$. A comunicação e o relacionamento estabelecidos na adoção das terapias complementares nos tratamentos contribuem para aumentar a satisfação do paciente com o tratamento e desempenha um papel importante na medida em que propiciam uma expectativa de resultado mais positivo ${ }^{23}$. 
As terapias alternativas/complementares são uma realidade para a amostra estudada, o que nos remete a alguns aspectos importantes que merecem ser considerados pelos profissionais, pelos serviços de saúde e convênios: 1) a existência de público interessado em tratamentos ainda não oferecidos por grande número de instituições de saúde, lembrando que as TAC podem ser utilizadas na promoção da saúde, o que configura um mercado potencial e pouco explorado; 2) o pouco envolvimento dos profissionais de saúde na indicação segura de tais práticas, o que pode comprometer a segurança do cuidado (o que requer investimentos em educação e qualificação); 3) a necessidade de estudos para ampliação da utilização das TAC fundamentadas em evidências científicas; e 4) a necessidade de avaliação de custos de tais práticas, uma vez que mesmo quem é atendido pela saúde suplementar (o que já caracteriza maior poder aqui-

\section{CONFLITOS DE INTERESSE}

declararam não haver

\section{FONTES DE FINANCIAMENTO}

nenhuma

\section{REFERÊNCIAS}

1. Virtual B. Medicina Complementar e alternativa 2008 [cited 2012 ; Available form: http: www.biblioteca virtual. Sp.gov.br especial 200804 - medicina complementar: php]

2. National Center for Complementary and Alternative Medicine. 2012 [cited July, 08, 2013]. Available from: http: http://nccam.nih.gov/health

3. Yates JS, Mustian KM, Morrow GR el al Prevalence of complementary and alternative medicine use in cancer patients during treatment. Support Care Cancer. 2005 Oct; 13(10): 806-11.

4. Salles LF, Silva MJP. Enfermagem e as práticas complementares de saúde. São Caetano (SP): Yendis; 2011.

5. Trovo MM, Silva MJP. Alternative complementary treatments - the view of nursing students. Rev Esc Enf USP. 2002 ; 36(1): 80-7.

6. Alfano ACC. Padrão do uso de terapias alternativas complementares por pacientes com câncer de mama metastático em quimioterapia e sua influência na qualidade de vida. [Dissertação]. Barretos (SP): Hospital do Cancer; 2013.

7. Molassiotis A, Fernandez-Ortega P, Pud D, Ozden G, et al. Use of complementary and alternative medicine in cancer patients: a European survey. Ann ONcol. 2005 Apr; 16(4): 655-63.

8. Spadacio C, Barros NF. Use of complementary and alternative medicine by cancer patients: systematic review. Rev Saude Publica. 2008 Feb; 43(1): 158-64.

9. Salles LF, Kurebayashi LFS, Silva MJP. As práticas complementares e a Enfermagem. In: Salles LF, Silva MJP. Enfermagem e as práticas complementares de saúde. São Caetano do Sul (SP): Yendis; 2011. Cap. 1, p.1-17. sitivo dessa clientela) considera o custo elevado com um impeditivo à continuidade dos tratamentos, bem como o que isso representa para o Sistema Único de Saúde com sua crescente e necessária adoção.

\section{CONCLUSÃO}

Parcela expressiva dos participantes, em sua maioria, mulheres adultas, referiu já ter utilizado algum tipo de TAC, sendo a acupuntura a mais citada. A experiência anterior positiva e o fato de ser um tratamento não medicamentoso foram os motivos mais citados para utilização da TAC em detrimento da orientação específica de um profissional de saúde. A perspectiva de novas formas de tratamento aliada à observação de resultados satisfatórios figuraram como importantes razões para adesão a este tipo de tratamento e a solução da queixa, bem como o custo elevado foram apontados para a não continuidade de uso das terapias complementares.
10. Brasil. Ministério da Saúde. Secretaria de Atenção à saúde. Departamento de Atenção Básica. Política Nacional de Práticas Integrativas e Complementares no SUS - PNPIC - SUS/ Ministério da Saúde, Secretaria de Atenção á Saúde, Departamento de Atenção Básica. Brasília: Ministério da Saúde; 2006.

11. Brasil. Ministério da Saúde. Portaria no 971, de 3 de maio de 2006. Diário Oficial da União, Brasília, 04 mai. 2006. Seção 1, p. 20-5.

12. Brasil. Ministério da Saúde. Secretaria de ciência, Tecnologia e Insumos Estratégicos, Departamento de Ciência e Tecnologia. Brasília: Editora do Ministério da Saúde; 2005.

13. Tsuchiya KK, Nascimento MJP. Terapias complementares: uma proposta para atuação do enfermeiro. Rev Enferm UNISA 2002; 3: $37-42$.

14. Nunez HMF, Ciozak Sl. Terapias alternativas/complementares: o saber e o fazer das enfermeiras do distrito administrativo $71-$ Santo Amaro - São Paulo. Rev Esc Enferm USP 2003; 37(3):11-8.

15. Souza VT. Enfermeiros que trabalham com terapias complementares: conhecendo sua prática. [Dissertação] São Paulo (SP): Escola Paulista de Medicina da UNIFESP; 2000.

16. Fontanella F, Speck FP, Piovezan AP, Kulkamp IC. Conhecimento, acesso e aceitação das práticas integrativas e complementares em saúde por uma comunidade usuária do Sistema Único de Saúde na cidade de Tubarão/SC. ACM arq. catarin. med. 2007; 36(2): 69-74.

17. Pinheiro RM, Castro CG Silva HH, Nunes JMG. Comportamento do consumidor e pesquisa de mercado. Rio de Janeiro: Editora FGV, 2006. 
18. Gonçalves DIF. Pesquisas de marketing pela internet: As percepções sob a ótica dos entrevistados. Revista de Administração Mackenzie 2008; 9(7): 70-88.

19. Dantas F, Tanigawa RY, Yamamura Y. Acupuntura. In: Diagnóstico e Tratamento. Barueri (SP): Manole; 2006. Cap. 3, p.422-30.

20. Jaconodino CB, Amestoy SC, Thofehrn MB. A utilização de terapias alternativas por pacientes em tratamento quimioterápico. Cogitare Enferm 2008; 13(1): 61-6.

21. Spadacio C, Barros NF. Uso de medicinas alternativas e complementares por pacientes com câncer: revisão sistemática. Rev Saúde Pública 2008; 42(1):158-64.
22. Spadacio C, Castellanos MEP, Barros NF, Alegre SM, Tovey P, Broom A. Medicinas Alternativas e Complementares: uma metassíntese. Cad. Saúde Pública 2010; 26(1): 7-13.

23. Buzato A, Kunzi B. Differences in the quality of interpersonal care in complementary and conventional medicine. BMC Complement Altern Med 2010; 10 (63): 1-14 